\title{
Paper of the August Issue of Stem Cell Reviews and Reports Reveals a Novel Role of Bone Marrow Stroma Cells Expressed CXCR4 in Preventing Aging of Hematopoietic Stem/Progenitor Cells
}

\author{
Mariusz Z. Ratajczak ${ }^{1}$ \\ Published online: 26 June 2020 \\ (C) Springer Science+Business Media, LLC, part of Springer Nature 2020
}

Beginning with this current August issue of Stem Cell Reviews and Reports, the Editorial Team will select and highlight the best paper in a given issue. This month we have selected the work of Dr. Pratibha Singh in the group of Dr. Louis Pelus, entitled "Aging-Related Reduced Expression of CXCR4 on Bone Marrow Mesenchymal Stromal Cells Contributes to Hematopoietic Stem and Progenitor Cell Defects".

Aging is an inevitable consequence of life and it has been suggested that it is preprogrammed in the genes of all living organisms. It is well known that aging accelerates after achieving reproductive age, when the genes could be passed on to the next generation. Several mechanisms are currently proposed that accelerate this process, leading to the same result of culling adult organisms that have completed the reproductive period of life. The hematopoietic system is not an exception to this rule and the aging process has negative effects on hematopoietic stem/progenitor cells (HSPCs). Aging impairs the regenerative potential of these cells and skews their differentiation towards the myeloid lineage. The age-associated pathophysiological changes of the hematopoietic system include the onset of anemia and diminished adaptive immune competence, and may lead to development of myelodysplasia and leukemia, all of which can be explained by the loss of normal homeostatic controls.

The bone marrow (BM) microenvironment and changes in hematopoietic niches has recently been suggested to influence HSPC aging, however the mechanisms whereby BM stromal cells mediate this effect is still not very well known. In the current interesting paper, Sing et al. focus on the consequences of an age-related decrease in expression of the CXCR4 receptor on BM stroma cells. As is well known, the

Mariusz Z. Ratajczak

mariusz.ratajczak@louisville.edu

1 University of Louisville, Louisville, KY, USA seven transmembrane spanning G $\alpha$ i protein-coupled chemokine receptor CXCR4 is expressed on hematopoietic stem/progenitor cells and its specific ligand $\alpha$-chemokine stroma derived factor-1 (SDF-1) plays an important role in embryonic development of hematopoiesis, and regulates BM retention, trafficking and survival of HSPCs in adults. However, in parallel CXCR4 is also present on bone marrow stroma cells.

In a set of elegant experiments, Sing et al. show that agerelated decrease in CXCR4 expression contributes to hematopoietic aging. Mechanistically, what was nicely shown in their paper, is that proper CXCR4 signaling prevents BM stroma cell dysfunction by suppressing oxidative stress and generation of reactive oxygen species (ROS). To support this, treatment of old or CXCR4 deficient BM stroma cells with the antioxidant $\mathrm{N}$-acetyl-L-cysteine (NAC), improved BM niche supporting the activity of these cells, and attenuated the aging phenotype of HSPCs. Similarly, treatment of CXCR4 deficient mice that show an aging HSPC phenotype with NAC largely reversed the aging phenotype.

The authors conclude that modulation of the SDF-1CXCR4 axis in bone marrow microenvironment may lead to novel interventions to alleviate the decline in the above mentioned immune/hematopoietic function that is seen with advanced age. This is important translationally because NAC is an off the shelf widely available anti-aging compound and based on the presented data could be easily employed as a nutritional diet supplement to positively modulate hematopoiesis and prevent aging related changes in the HSPCs compartment.

Publisher's Note Springer Nature remains neutral with regard to jurisdictional claims in published maps and institutional affiliations. 